\title{
Symbolic Trajectory Description in Mobile Robotics
}

\author{
Pradel Gilbert¹, Căleanu Cătălin-Daniel² \\ (1)IBISC laboratory, \\ University of Evry Val d'Essonne \\ 40 Rue du Pelvoux, 91020 Evry cedex, France \\ (2) Electronics and Telecommunication Faculty \\ Politehnica University of Timisoara \\ Bd. Vasile Parvan, 2, 300223 Timisoara, Romania
}

\section{Introduction}

One main issue for mobile robots is their capacity to go from one point to another autonomously, without getting lost or crashing into another object (Arkin, 1998).

It is based on three concepts:

- planning which computes a trajectory between the two points,

- navigation which gives motion orders to the robot to follow the computed trajectory,

- environment representation which permits the robot to know if it goes in the right direction.

Works presented here are interested in point 3, that is, in acquiring spatial models of the robot's physical environment.

Two different approaches to this problem have emerged. The first one, the metric/quantitative representation of the environment, has some disadvantages. For example, due to incorrigible wheel slippage, dead-reckoning could be unreliable. The nonmetric/qualitative approach use perceptual landmarks to generate maps and to localise the robot with respect to these landmarks. Works presented here are interested in the nonmetric approach, trying to perform a qualitative description of a stuctured indoor environment.

These problems are tackled by the Simultaneous Localisation And Mapping (SLAM) introduced by Leonard and Durrant-Whyte (Leonard \& Durrant-Whyte, 1991) (Smith \& Leonard, 1997) in robotics. SLAM is still today a very active field of research (Meyer \& Filliat, 2002)(Filliat \& Meyer, 2002a,b). This problem is regarded as one of most significant for a true autonomy of the robots. Crucial questions still remain satisfactorily unanswered in spite of great progress in this field and the existence of robust methods to map static, very structured and limited sized environments.

(Kulic and Vukic, 2003) use a robot motion planning based on behavioural cloning. In a first phase, the robot is trained under operator's control to locate unmoving obstacles avoidance through a simulator. In that phase, the evaluated variables are stored in a log file. The 
second phase, called learning phase, machine learning program generates the differential equations defining the operator's trajectory, i.e. the clone. Finally, the verifying phase, the robot is controlled by the clone. These developmental phases are repeated changing both problem domain representation and learning system according to the cloning system criterion.

The problem of mapping can be generally regarded as the fact of giving to an autonomous robot the capacity to move in an environment. Thus, the problem of mapping goes further than simple construction of a plan gathering the obstacles in a given zone. Thrun (Thrun, 2002) gives a general survey of the mapping problem. He points out the six key aspects of the mapping problem:

- The effects of the noise in the measurements (Wheel slippage, localisation error introduced by integration of data from wheel encoders, drift of inertial systems are three examples among many others.),

- The high dimensionality of the entities that are being mapped (How many parameters describe the environment, its major topological elements like corridors, crossings, doors, rooms, etc.?),

- The correspondence problem, also known as the data association problem (Do the measurements made by the sensors at different points in time in the environment correspond to the same object?),

- The perceptual aliasing (Two different places from the environment can be perceived in an identical way by the sensors.),

- The environment changes over time,

- The robotic exploration, that is the task of generating robot motion in the pursuit of building a map.

This chapter is organised as follow. Section 2 gives an overview of the works in the field of environment representation. Section 3 briefly presents the test-bed perception system. Sections 4 and 5 detail our approach in the digitised construction of the environment from the distance measurements, the extraction of the landmarks and explain the fresco construction and its validation. In section 6, we propose a method to represent robot's trajectory based on series of landmarks called frescoes and different methods to select the most salient of them with which it is possible to describe the environment. Section 7 shows and discusses the experimental results. We conclude with ways to improve the method.

\section{Related works}

Related works can be found in the fields of Image Based Navigation systems, shape understanding using sensor data, vision based homing. Vision for mobile robot navigation did have specific development during the last twenty years. (DeSouza \& Kak, 2002) give a complete survey of the different approaches. For indoor navigation, systems are classified in three groups: map-based navigation using predefined geometric and/or topological models, map-building-based navigation constructing by themselves geometric and/or topological models, and mapless navigation using only object recognition and actions associated to these objects (Gaussier \& al. 1997).

Kuipers' works (Kuipers \& Byan, 1991) defined symbols as distinct places situated at equal distances from the nearby obstacles. Connections between these places link symbols and represent free path (Choset \& Nagatani, 2001). Fig. 1 shows an example of the Voronoii 
graph of an environment. The labelled vertices represent the symbols while edges connecting the symbols are the path the robot can use.

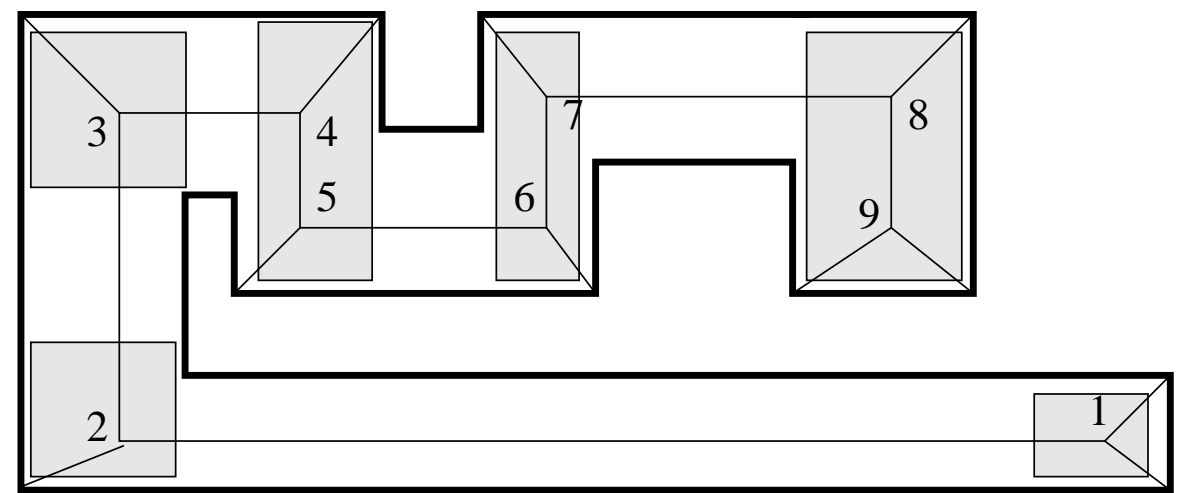

Fig. 1. Voronoii diagram with labelled vertices.

Indeed, assume that the robot has to move in this simple environment (Fig. 1) according to a mission given by the user, if the robot goes from label 1 to label 9, the most important areas are those filled, where the robot changes its direction. Between them, when there are no changes in the environment, it is useless to preserve the whole set of information from this part of the way. On the contrary, it is necessary to find a method of mapping of the filled zones which can describe them unambiguously.

In Image Based Navigation systems, several great classes of systems can be identified from the literature. The first one uses conventional telemeters and vision to find and identify objects in the environment (Wichert, 1996). The second one is the class of the systems coupling more or less directly sensor data to motor control thanks to a supervised learning process. Among them neural networks systems used as classifiers are noticeable. These systems begin to classify the environment into global classes such as "corridor, corner, room, crossing ..." (Al Alan \& al. ,1995) (Pomerleau, 1993) are often followed by a second processing unit that outputs a navigation command. In addition to restrictions related to the supervised learning, these classes give only a global description and are of least interest in cluttered and complex environments. The third class includes the systems which compare current sensor data and predefined models both at a low level (edges, planes ...) - see (Kim \& Neviata, 1994) - and at a high level (door, room, object ...). These systems use mainly vision sensors (cameras) that provide a huge amount of data that must be reduced to be processed in real time. The elements extracted from the data are compared to reference models known a priori. The fourth class evoked here includes the systems trying to geometrically build environment models before deciding an optimised path plan (Crosnier, 1999).

In the field of shape understanding using sensor data, environment interpretation stresses the use of natural landmarks to ease the navigation and the pose estimation of a mobile robot. Among other works, one can pinpoint (Simhon \& Dudek, 1998a) which is interested in defining islands of reliability for exploration. He proposes strategies to couple navigation and sensing algorithms through hybrid topological metric maps. (Oore \& al., 1997) consider the problem of locating a robot in an initially unfamiliar environment from visual input. In the same way, (MacKenzie \& Dudek, 1994) involve a methodology to bind raw noisy sensor data to a map of object models and an abstract map made of discrete places of interest. 
Several implementations of vision based homing systems are presented in (Franz \& al., 1997). A method aiming at highlighting salient features as, for example, landmarks between these two views and deriving a decision is used in (Hong, 1991). In these works, a homing system extracts landmarks from the view and allows a robot to move to home location using sequence target locations situated en route between its current location and home. Other works are biologically inspired. (Judd \& Collett, 1998) showed that ants store series of snapshots at different distances from their goal to use them for navigating during subsequent journeys. Judd and Collett experimented their theory with a mobile robot navigating through a corridor, homing successive target locations. Weber (Weber \& al., 1999) proposes an approach using the bearings of the features extracted of the panoramic view leading to a robust homing algorithm. This algorithm pairs two landmarks situated into two snapshots to derive the homing direction. The bearings-pairing process uses a list of preferences similar to neighbourhood rules.

Symbolic processing methods are described in Tedder's works (Tedder \& Hall, 2001). This formal approach is often called structural or syntactic description and recognition. The general method for perception and interpretation proposes to symbolically represent and manipulate data in a mapping process. (Tedder \& Hall, 2001) solve the problem in modelling the 3D environment as symbolic data and in processing all data input on this symbolic level. The results of obstacle detection and avoidance experiments demonstrate that the robot can successfully navigate the obstacle course using symbolic processing control. These works use a laser range finder. A way for defining suitable landmarks from an environment as the robot travels is a research problem pointed out by Fleisher and al. in (Fleisher and al., 2003). An automatic landmark selection algorithm chooses as landmarks any places where a trained sensory anticipation model makes poor predictions. The landmark detection system consists of a sensory anticipation network and a method of detecting when the difference between the prediction of the next sensor values and the current measured values can reveal the presence of a landmark. This model has been applied to the navigation of a mobile robot. An evaluation has been made according to how well landmarks align between different runs on the same route. These works show that the robot is able to navigate reliably using only odometry and landmark category information.

In (Lamon \& al., 2001), a method is proposed for creating unique identifiers called fingerprint sequences for visually distinct significant features in panoramic images. This localisation system proves that the actual position of a robot in an environment can be recovered by constructing a fingerprint sequence and comparing it with a database of known fingerprints.

The proposed work goes on the way proposed by (Tedder \& Hall, 2001) and (Lamon \& al., 2001). According to these works, our contribution applies mainly on a method to extract clues of interest among raw distance data delivered by a $2 \mathrm{D}$ panoramic laser range finder installed on the robot. These clues of interest, i.e. the landmarks, are gathered in a sequence that we call a fresco. We consider that the trajectory of the robot can be described by the set of the frescoes. To do that, we have to select the frescoes that bring new information. The originality of this work stays in the simple but efficient criteria used for the construction and the validation of the fresco but mainly to select the most pertinent frescoes along the route of the robot. In addition to this qualitative approach, one must consider that the system will have to be embarked on a vehicle, which vibrates, runs at variable speeds on a non-uniform ground. This leads to constraints of speed, size, robustness, compactness and cost, implying 
various choices both at the design and at the development levels of the system. The methods used have been chosen as simple as possible to reduce the cost and the complexity of the processing. Nevertheless the method must be robust compared with the robot movements, the sensor accuracy and the variations of the complexity of the environment.

\section{Test-bed perception system}

The application field of this work is a middle-cost mobile robot sent in an apartment to do service for a user. Hence, the environment is of a structured and not engineered indoor type environment. At this point, the problem is two-fold. Firstly, through the Human-Machine Interface (HMI), a mission must be entered and its development must be explained to the user. Secondly, the robot has to be programmed to execute the mission. Building a description of the route as close as a human could do has at least two advantages. This description, on one hand, is requested by the HMI and, on the other hand, at the execution level, it can be a way to take into account the stumbling blocks highlighted by the conventional navigation systems.

The size of the non holonomous robot is (width $\times$ length) $0.50 \mathrm{~m} \times 0.75 \mathrm{~m}$. Its linear and angular speeds are up to $1 \mathrm{~ms}^{-1}$ and $2.45 \mathrm{rads}^{-1}$. Placed at the geometrical centre of the robot with practical/maximum ranges equal to $3 \mathrm{~m} / 10 \mathrm{~m}$, a panoramic $2 \mathrm{D}$ telemeter captures a circular environment. It has been decided to consider a $36 \mathrm{~m}^{2}$ squared environment to ease the reconstruction process (measurements at the corners are valid according to the maximum range of the telemeter). Only 256 measurements over the 1024 the telemeter is able to deliver are used by the fresco construction process. At a $1 \mathrm{~ms}^{-1}$ speed, the translation displacement error remains lower than $10 \mathrm{~cm}$ for one complete rotation of the telemeter. In $100 \mathrm{~ms}$, the rotation of the robot remains lower than $23^{\circ}$. Experiments in the following have been made with measurements coming from both a simulated laser range finder and the real telemeter.

We will then consider that:

- there is a lack of accuracy of the telemetry measurements due to the vibrations caused by the jolts,

- most part of the environment is composed of co-operative targets (low material absorption coefficient, acceptable level of the reflected signal up to a $80^{\circ}$ incident angle),

- reference position of the laser coincides with the main axis of the robot,

- data sequencing compensates the effects of the clockwise (CW) or counter clockwise $(\mathrm{CCW})$ rotations of the robot so that the 256 horizontal distance information are regularly arranged on $360^{\circ}$,

- $\quad$ precision is greater than $20 \mathrm{~cm}$ for every measurements.

According to these considerations, we chose to digitise the environment on a $32 \times 32$ cells grid which covers the area seen by the telemeter, each cell representing a $0.1875 \mathrm{~m} \times 0.1875 \mathrm{~m}$ square. The terms "grid" or "cellular space" will be considered as equivalent in the following.

\section{Representation construction}

\subsection{Cyclic representation and cellular space}

Landmarks such as "Opening, Closure, End_of_Closure, Angle_of_Closures" used to build the qualitative description of the environment from the measurements. According to the 
sequential aspect of the data delivered by the laser range finder, the landmarks extraction order corresponds to the measurements order. The robot refers to two main axis: the "lengthwise axis" corresponds to the forward and rear directions of displacement, the "crosswise axis" is perpendicular to the lengthwise axis at the robot geometrical centre.

The fresco construction is divided into two main steps:

- The construction of the reliable digitised environment: cellular space building, signature extraction, crosswise, lengthwise and diagonal segments extraction, refining, reorientation.

- The landmarks extraction: Opening, Closure, End_of_Closure and Angle_of_Closures extraction, fresco construction, fresco validation.

The cellular space appears in fig. 4 a.

\subsection{Conventions used in the cellular space}

The method uses evolution laws in the cellular space that act on every cells. For a cell called CELL the neighbourhood conventions use standard Von Neuman neighbourhood. For example, CELL_W, CELL_E, CELL_N, CELL_S are the names of the cells situated westbound, eastbound, northbound, southbound. We add the word Great to name the cells in the second neighbourhood layer (Great West: CELL_GW, Great East: CELL_GE ...). The quadrants are numbered counter clockwise in relation to the lengthwise axis: quadrant 0 is the front right one.

\subsection{Construction of the digitised description}

Fig. 2 summarises the operations leading to the construction of a reliable cellular space (Pradel and al., 1994).

(a) Generation of the digitised environment: the very first operation performed consists in the lay-down of the distance measurements onto the grid to create the initial cellular spaces. They perform the same operations on the distance measurements issued from the sensor (part 1) and on the $45^{\circ}$ shifted measurements set (part 2). On the grid, black cells represent the range finder impacts. Noise introduced in the measurements (measurements are made while the robot is moving) appears mainly on the form of cells agglomerations (fig. 4a). Agglomerations also occur when measurements belong to the border between adjacent cells. Elimination of agglomerations (fig. 4a, b) is performed keeping only the cells situated the closest to the robot for obvious safety reasons. The method adopted for this elimination uses evolution laws close to those used in cellular automata.

(b) Segmentation of the cellular space: the next operation is the extraction of the segments corresponding to the obstacles from the cellular space. Four directions are considered. In addition with the lengthwise (fig. 3a) and crosswise axis (fig. 3c), a search for the segments is made onto the two diagonals (fig. $3 \mathrm{~d}, \mathrm{f}$ ). The extraction laws leave alive a cell owning a neighbour alive in the considered direction.

(c) Reorientation of the cellular space: as shown in fig. 4a, another origin of noise is bound to the oblique walls. These digitised oblique walls take the form of small adjacent segments with junctions without real significance. To eliminate these oblique walls and the noise they introduce we decided to use a second grid on which the measurements are laid with a $45^{\circ}$ angular shift (Part 3). Superfluous data elimination and segmentation are also applied on this second grid. 


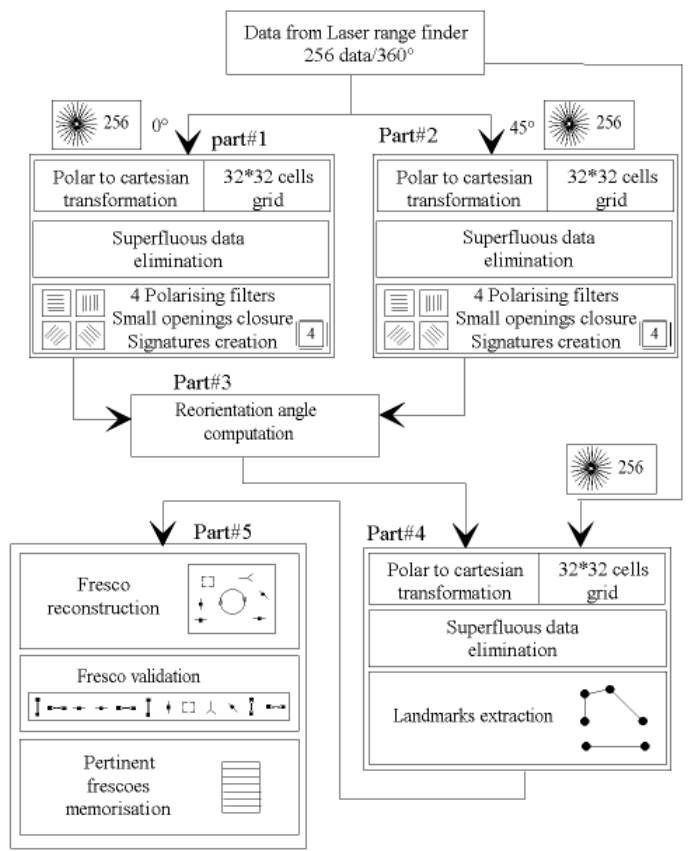

Fig. 2. lock diagram showing the operations performed in the construction of the digitised environment.
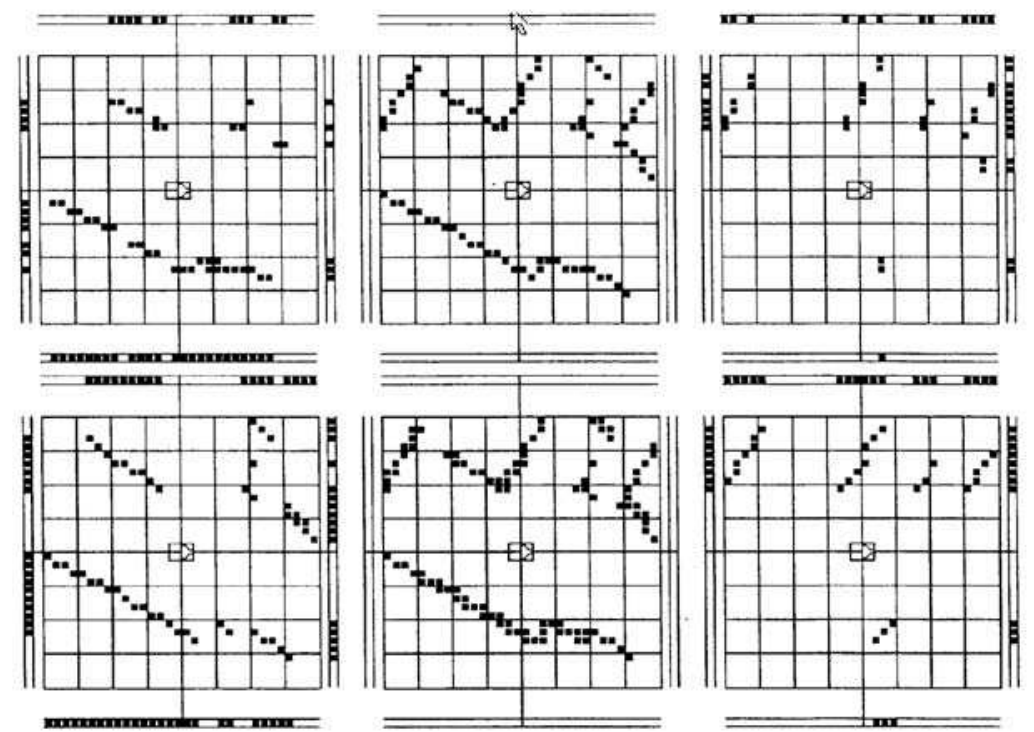

Fig. 3. Extraction of segments in the 4 filtering directions: a (upper left): Lengthwise segmentation, b (upper centre): Refined environment, 
c (upper right): Crosswise segmentation,

d (lower left): First diagonal segmentation,

e (lower centre): Initial measurements,

$\mathrm{f}$ (lower right): Second diagonal segmentation

A search for the longest and the shortest continuous segments is performed (Bras \& al., 1995) among the projections of the environment on the crosswise and lengthwise axis in each quadrant of the cellular space according to a filtering direction (lengthwise, crosswise, diagonal 1 and 2). A reorientation angle is then computed according to:

$$
\Theta=\arctan \frac{\text { ShortestCrosswiseSegment }}{\text { LongestLengthwisesegment }} \pm n * \frac{\pi}{4}
$$

According to the direction in which the longest segment is found (i.e. the most plausible reference in the environment), adequate choices for the sign and the value of $\mathrm{n}(\mathrm{n}$ in $\{0,1\})$ lead the robot to be reoriented parallel to the longest segment $(0 \leq \Theta \leq \pi / 3)$ or perpendicularly to it $(\pi / 3<\Theta \leq \pi / 2)$. The reoriented cellular space is re-built from the initial measurements according to the reorientation angle. Fig. $4 \mathrm{c}$ shows the benefits of the reorientation. The reoriented cellular space is then considered as reliable and will allow the landmarks to be extracted.

(d) Landmarks extraction: as told in the introduction, the environments are described using a fresco made of ordered series of landmarks: "Opening", "Wall" also called "Closure" and "Corner" also called "Angle_of_Closures". Let us note that an "Angle_of_Closures" must be neighboured by two "End_of_Closure" landmarks. The landmarks extraction first considers the "Opening" elements that are directly extracted from the reoriented signatures.

The "Angle_of_Closures" and "End_of_Closure" landmarks are extracted from the reoriented cellular space by the following laws. The first operation consists in the "Angle_of_Closures" extraction by the following equation that is applied to every cell in the grid:

$$
\begin{aligned}
& \text { Angle_of_Closures }=((\text { CELL \& CELL_W }) \mid(\text { CELL \& CELL_E })) \&((C E L L ~ \mid \\
& \text { CELL_N }) \mid(\text { CELL \& CELL_S })) \& \text { neg CELLdiag }
\end{aligned}
$$

with: CELLdiag meaning that the logical state of the cell is true if it belongs to a diagonal. Operators \& (logical AND) and neg (logical NOT) are applied on the states of the cells.

The first ligne of this equation checks if the cell has east or west neighbours while the second line checks north and south neighbours. Therefore a cell is considered as an Angle_of_Closures if it has at least a crosswise and a lengthwise neighbour.

The second operation aims at extracting the "Lenghtwise End_of_Closure" and "Crosswise End_of_Closure" landmarks. These operations are allowed if and only if the cell does not belong to the two diagonals and is not an "Angle_of_Closures".

Fig. 4d and 4e show the "Angle_of_Closures" and "End_of_Closure" landmarks positioned on the grids. To each landmark are associated three qualitative attributes representing three properties of landmarks. The off-sight attribute is set when the landmark stands on the cellular space border. The position attribute can take the following values: crosswise, diagonal, lengthwise according its position. The certainty attribute is introduced to take into account landmarks that could come from a possible noise introduced in the digitisation process not detected by the previous laws or a still possible bad reorientation. It is false for every landmark (for instance, diagonal "End_of_Closure", " $45^{\circ}$ _angles") whose evolution cannot be known. 


\section{Fresco construction}

The first step of the fresco construction gathers the landmarks space into ordered series of semantic clues and describes the environment by positioning landmarks in respect to each others. Each landmark has exactly two neighbours (the last landmark in the list has the first one as second neighbour). Building the fresco is made using the symbols presented in Table 1 which gathers the landmarks identity and attributes. The landmarks identity and attributes have been chosen according to the indoor environment in which the robot moves. This operation mainly aims at eliminating the notion of distance to the profit of a spatial series and highlights the qualitative representation of the environment. An example of fresco is given in fig. 4f. The robot is situated in the middle of the environment. To each landmark are associated three qualitative attributes representing three properties of landmarks. The off-sight attribute is set when the landmark stands close to or beyond the end of the sensor range. The position attribute can take the following values: crosswise, diagonal or lengthwise according its position related to the lenghtwise and crosswise robot axis. The certainty attribute is introduced to take into account landmarks whose evolution can be forecast. It is false for every landmark (for instance, diagonal "End_of_Closure", " $45^{\circ}$ _angles") that could come from a possible noise introduced in the digitisation process and whose evolution cannot be known (Pradel \& al., 2000), (Pradel \& Bras, 2001).

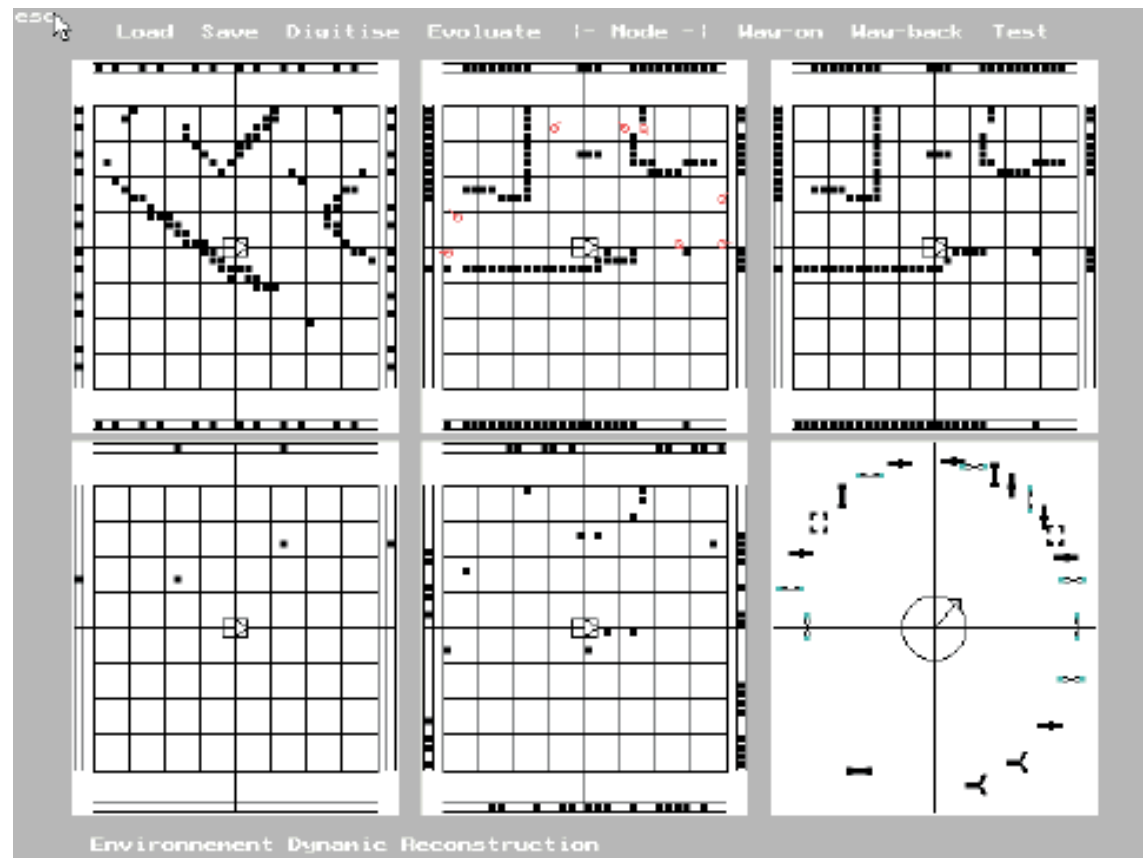

Fig. 4. Example of the digitised constructions:

a (upper left): real world from raw measurements; b (upper centre): reoriented cellular space; c (upper right): refined space after superfluous data elimination;

d (lower left): Angles_of_Closure extraction, e (lower centre): End_of_Closure extraction; f (lower right): fresco construction 


\begin{tabular}{|c|c|c|c|c|}
\hline Symbol & Landmark & Position & Off-sight & Certainty \\
\hline 7 & Angle_of_Closure & & & True \\
\hline & End_of_Closure & lengthwise & & True \\
\hline & End_of_Closure & lengthwise & off_sight & False \\
\hline & End_of_Closure & crosswise & & True \\
\hline & End_of_Closure & crosswise & off_sight & False \\
\hline 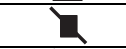 & End_of_Closure & diagonal1 & & False \\
\hline & End_of_Closure & diagonal1 & off_sight & False \\
\hline $\boldsymbol{H}$ & End_of_Closure & diagonal2 & & False \\
\hline 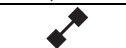 & End_of_Closure & diagonal2 & off_sight & False \\
\hline$<$ & $45^{\circ}$ Angle & lengthwise & & False \\
\hline$\lambda$ & $45^{\circ}$ Angle & crosswise & & False \\
\hline$\infty$ & Opening & lengthwise & & True \\
\hline $\bar{X}$ & Breakthrough & lengthwise & & True \\
\hline & Opening & crosswise & & True \\
\hline$\approx$ & Breakthrough & crosswise & & True \\
\hline
\end{tabular}

Table 1. Landmarks used in the fresco construction.

The second step focuses on the fresco validation. Assuming that there is only one description for one environment, strict laws of neighbourhood are defined. Fig. 5 shows these neighbourhood laws that can be interpreted as a set of logical assertions. An Angle_of_Closure can only have as neighbours Angle_of_Closures or End_of_Closures. For each landmark, the neighbourhood is checked. Every time a fresco is built, the whole set of these rules is applied in order to validate the fresco. If one rule failed, the fresco is not valid.
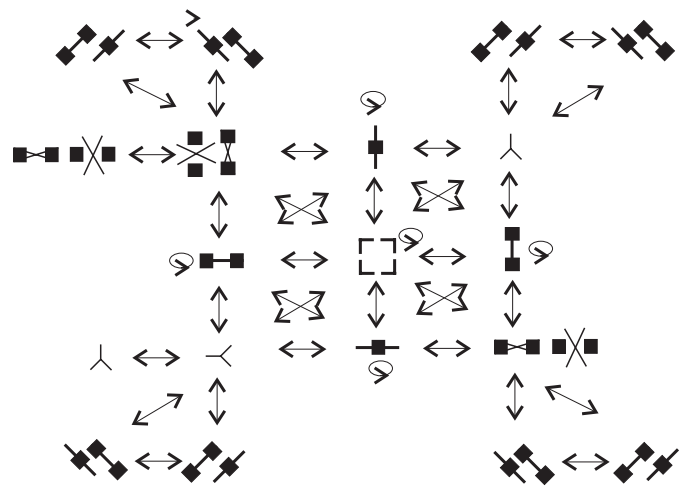

Fig. 5. Landmarks neighbourhood rules.

The validation fails mainly due to a bad landmark extraction process in a very noisy cellular space or a bad reorientation. Making the necessary corrections in the extraction laws to solve these seldom failing cases leads to an increasing of the complexity of the evolution laws, increasing not really justified by the low frequency of the failures. We consider that the loss of a fresco is not an important drawback: a failure in the validation of the fresco will 
be corrected by the next valid one with only slight effects on the mission of the robot and the effect of this loss is very attenuated because the process of transitions detection and environment memorisation eliminates a greater part of the frescoes. When it is validated, the fresco appears as shown in fig. 4f. A fresco will contain at most 64 landmarks symbols organised into 4 sectors of 16 symbols at most.

\section{Symbolic trajectory description using frescoes}

Building the symbolic description of the route followed by the robot is three-fold:

- how to build the qualitative descriptions (frescoes) in accordance with the robot's sensors?

- $\quad$ how to describe the route by a sequence of the most pertinent frescoes?

- how to use these frescoes with the control-command level of the robot?

This section deals with the second point. The choice of the most salient frescoes is made using different criteria described in the following sections. Every time the laser range finder scans the environment, a fresco is built. In our case, the fresco built-in period is $300 \mathrm{~ms}$. Hence, if all frescoes are stored their number grows quickly and some of them are not useful. Storing all the frescoes when the robot runs in a corridor is a trivial example. All frescoes are very similar excepted at both ends. If only few frescoes are useful, how then is it possible to select them? Is a specific sequence of frescoes able to describe a part of the environment? Answering, at least partially, to these questions is the aim of this section.

Following a specific path, the total number of stacked frescoes could be large enough. Moreover, successive frescoes could be identical or slightly different. Therefore, a selection of meaningful frescoes, which offers a thoroughly environment description, is absolutely necessary. Based on these salient selected frescoes, the robot also should be able to find a return path. In local homing for example, an agent returns to a previously visited location by moving to maximize the correspondence between what it sees currently and a remembered view from the target.

In dealing with frescoes, which are basically a collection of symbolic strings, we were inspired by different methods, such as those used in spell checking, optical character recognition (OCR), molecular biology for DNA or amino-acid sequences study (Altschul, 1991), (Karlin, 1990) or computational intelligence.

The first two criteria proposed to evaluate a kind of distance between frescoes are called resemblance and barycentre. A new fresco is considered as bringing new information if its distance to the previous stored one regarding one of the criteria is greater than a threshold. The two next sections describe these criteria. A systematic study gives an evaluation of the thresholds to use to make the criteria effective.

\subsection{Resemblance method}

This criterion uses a nearby principle of that presented in (Hong, 1991). A correlation function allows calculating the resemblance between two frescoes. This criterion has been tested in the same environment as that used for the construction and the validation of the frescoes. The use of this criterion shows that the landmarks that are not certain make very difficult the evaluation of the resemblance so only the certain elements were kept. The resemblance between two consecutive frescoes is calculated by taking into account the difference between the number of certain landmarks in the corresponding quadrants. The resemblance between two frescoes is calculated from the difference between the number of 
landmarks in respective quadrants of two consecutive frescoes. The comparison of this difference with a reference threshold indicates if the current fresco should be kept or rejected because not bringing enough information.

The resemblance between two consecutive frescoes $i$ and $j$ is calculated as:

$$
\mathrm{r}_{\mathrm{ij}}=\left|\mathrm{N} 0_{\mathrm{i}}-\mathrm{N} 0_{\mathrm{j}}\right|+\left|\mathrm{N} 1_{\mathrm{i}}-\mathrm{N} 1_{\mathrm{j}}\right|+\left|\mathrm{N} 2_{\mathrm{i}}-\mathrm{N} 2_{\mathrm{j}}\right|+\left|\mathrm{N} 3_{\mathrm{i}}-\mathrm{N} 3_{\mathrm{j}}\right|
$$

where

$\mathrm{Nk}_{\mathrm{i}}, \mathrm{k}=1 \ldots 4$ represents the number of landmarks in quadrant $\mathrm{k}$ of the $\mathrm{i}$-th fresco $\mathrm{Nk}_{\mathrm{j}}, \mathrm{k}=1$ ... 4 represents the number of landmarks in quadrant $k$ of the $j$-th fresco .

If the resemblance $r_{i j}$ is greater then an a priori specified threshold then the $j$-th fresco will be selected and memorized as sufficiently different from the rest.

\subsection{Barycenter method}

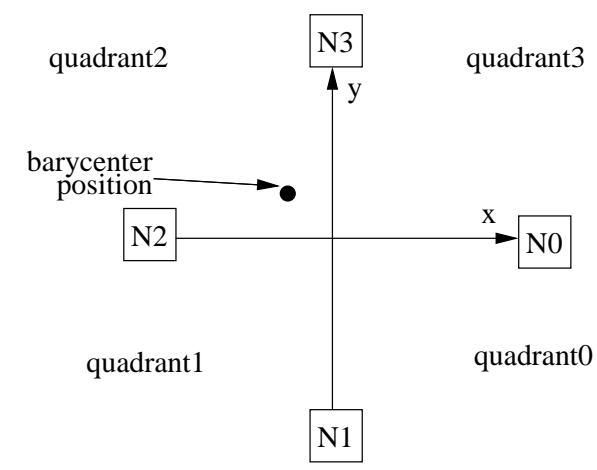

N0: number of certain landmarks in quadrant0

$\mathrm{N} 1$ : number of certain landmarks in quadrant1

$\mathrm{N} 2$ : number of certain landmarks in quadrant2

N3: number of certain landmarks in quadrant3

Fig. 6. Barycenter computation between certain landmarks.

This criterion is inspired by the distance of Hausdorff which measures the distance between two sets (Ahuactzin \& al., 1995), (Huttenlocher \& al., 1993). In our case, this notion was very simplified to respect real-time constraints. It takes into account only the number of certain landmarks in every quadrant. The landmarks are positioned as indicated on the fig. 6 and the barycentre is positioned at the following coordinates:

$$
\begin{aligned}
& x r e f=\frac{N 0_{i}-N 2_{i}}{N t_{i}} ; x=\frac{N 0_{j}-N 2_{j}}{N t o t_{j}} \\
& y r e f=\frac{N 1_{i}-N 3_{i}}{N t_{i}} ; y=\frac{N 1_{j}-N 3_{j}}{N t o t_{j}} \\
& \text { bary }_{i j}=\sqrt{\left(x_{r e f}-x\right)^{2}+\left(y_{r e f}-y\right)^{2}}
\end{aligned}
$$

where

$\mathrm{Nk}_{\mathrm{i}}, \mathrm{k}=1 \ldots 4$ is the number of landmarks in quadrant $\mathrm{k}$ of the $\mathrm{i}$-th fresco,

$\mathrm{Nk}_{\mathrm{j}}, \mathrm{k}=1 \ldots 4$ is the number of landmarks in quadrant $\mathrm{k}$ of the fresco, 
Ntot $_{i}$ and Ntot $t_{j}$ are the total numbers of certain landmarks in the $i$-th $/ \mathrm{j}$-th frescoes respectively.

Any variation of the number of elements in a quadrant implies a movement of the barycentre. If this displacement is greater then an a priori specified threshold then the j-th fresco will be selected and memorized.

\subsection{Distances based methods}

Distance is usually, but not necessarily, defined on a vector space. For strings, there are also some ways for quantifying how much two strings differs, as we will see in the next sections. These metric functions attempt to ascribe a numeric value to the degree of dissimilarity between two strings.

(a) Hamming distance method: the Hamming distance (HD) could be defined only for strings of the same length (Gusfield, 1997). For two strings, $S_{1}$ and $S_{2}$, the Hamming distance $\operatorname{HD}\left(\mathrm{S}_{1}, \mathrm{~S}_{2}\right)$ represents the number of places in which the two strings differ, (Lamon, 2001) have different characters as shown in the following example: HD $\left({ }^{\prime} \mathrm{ABCD}^{\prime}\right.$, ' $\mathrm{ACDB}$ ') $=3$

(b) Levenshtein distance method: the Levenshtein distance (LD) realizes a more complex evaluation of two strings than the Hamming distance. It could operate with strings not necessary of the same length and represents the minimum number of elementary transformations (insertion, deletion and substitution of a symbol) needed to transform one string into another (Levenshtein, 1966):

$$
\mathrm{LD}\left(\mathrm{S}_{1}, \mathrm{~S}_{2}\right)=\min \left(\mathrm{N}_{\mathrm{ins}}+\mathrm{N}_{\text {del }}+\mathrm{N}_{\text {subs }} \mathrm{t}\right)
$$

Closely related to it is the weighted Levensthein distance (WLD) also known as edit distance, where different costs are assigned to each edit operation (Kohonen, 1988) (Wagner, 1974):

$$
\mathrm{WLD}\left(\mathrm{S}_{1}, \mathrm{~S}_{2}\right)=\min \left(\mathrm{w}_{\text {ins }} \mathrm{N}_{\text {ins }}+\mathrm{w}_{\text {del }} \mathrm{N}_{\text {del }}+\mathrm{w}_{\text {subst }} \mathrm{N}_{\text {subst }}\right)
$$

(c) N-Gram method: an N-gram is a substring of $\mathrm{N}$ consecutive symbols. Let $\mathrm{N}_{1}$ and $\mathrm{N}_{2}$ be the number of $N$-grams in strings $S_{1}$ and $S_{2}$, respectively let $m$ be the number of matching $N$ grams. If one string is longer than the other, the unmatched $\mathrm{N}$-grams are also counted as differences. The feature distance (FD) is defined then as (Kohonen, 1987):

$$
\mathrm{FD}\left(\mathrm{S}_{1}, \mathrm{~S}_{2}\right)=\max \left(\mathrm{N}_{1}, \mathrm{~N}_{2}\right)-m\left(\mathrm{~S}_{1}, \mathrm{~S}_{2}\right)
$$

\subsection{Similarity based methods}

Finding similarities in character strings is an important problem in text processing and data mining. It has applications in genetics research as well, since strands of DNA can be expressed as very long strings of the characters.

A similarity measure is simpler than a distance. For strings $S_{1}, S_{2}$, finding similarities in character strings is an important problem in text processing and data mining. It has applications in genetics research as well, since strands of DNA can be expressed as very long strings of characters.

A similarity measure is simpler than a distance. For strings $S_{1}, S_{2} \in S$, any function $s: S^{2} \rightarrow \Re$ can be declared similarity. For strings, similarity is closely related to alignment.

(a)Cross correlation matching method 
This function is commonly used in signal processing. For symbols, the function compares string $S_{1}$ (of length $\mathrm{m}$ ) with $\mathrm{S}_{2}$ (of length $1=\mathrm{n} \geq \mathrm{m}$ ) and produces a cross correlation similarity vector, CCS, of length $(1=m+n-1)$ with elements CCS $_{i}($ with $i=0,1 \ldots 1-1)$ given by (Gusfield, 1997) (Haykin, 1999):

$$
C C S_{i}\left(s_{1}, s_{2}\right)=\left\{\begin{array}{l}
\sum_{j=0}^{i} S\left(s_{1 j}, s_{2(n-1)-i-j}\right), \text { if } i=0 \ldots m-1 \\
\sum_{j=0}^{m-1} S\left(s_{1 j}, s_{2(n-1)-i-j}\right), \text { if } i=m \ldots n-1, m \neq n \\
\sum_{j=0}^{(l-1)-i} S\left(s_{1(n-1)-(l-1-i)+j}, s_{2 j}\right), \text { if } i=n \ldots l-1
\end{array}\right.
$$

where:

$$
S(x, y)=\left\{\begin{array}{l}
1 \text { if } x=y \\
0 \text { if } x \neq y
\end{array}\right.
$$

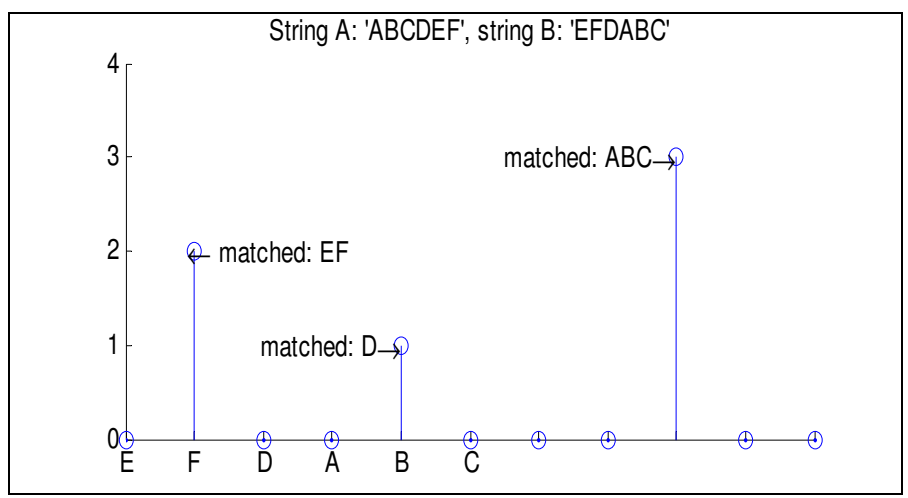

Fig. 7. Results of the cross correlation function. Peak value is obtained for the alignment of "ABC" tri-gram.

Fig. 7 gives an example of the results given by the cross correlation matching method for two strings of length equal to 6 .

\subsection{Neural network based method}

Speaking in a neural network terminology, finding the salient frescoes is equivalent with finding prototype vectors. Self Organizing Feature Map-Neural Networks, SOFM-NN, tries to place or adapt neurons in such a way that they serve as good prototypes of input data for which they are sensitive.

(a) Classic SOFM-NN: these networks are based on unsupervised competitive learning and winner-takes-all neurons (Haykin, 1999). During the training phase a SOFM-NN creates a topologic correspondence between the spatial location of the neurons in the output layer and the intrinsic features of the input patterns. If there is some similarity between input vectors then neighbours neurons will fire. If two input patterns are different than output 
neurons situated at considerable distance or spatial location will respond. For prototype vectors calculus usually Euclidian distance is used, as elements having the smallest sum of squared distance over the data set.

The principal problem is that classic SOFM-NN training algorithm is defined for numbers and not for strings. There are numerous ways for string to numbers conversion and vice versa (Aha \& al., 1991) (Blanzieri, 1999). For our particular case, the maximum number of symbols within a fresco is 16 , hex coded. So the NN input vector could be constructed by means of:

- Direct coding: each symbol had its own binary equivalent $(0=0000,1=00001 \ldots \mathrm{F}$ $=1111$ ),

- Exclusive coding that is, the symbol is coded with an unary vector with all the components but the $\mathrm{i}$-th set to zero $(0=000 \ldots 0001,1=00 \ldots 010, \ldots, \mathrm{F}=10 \ldots$ 000).

Finally, a fresco will be represented as a binary vector composed by concatenation of each binary coded constituent string.

(b) Symbolic SOFM-NN: based on distance measure for strings and calculating the prototype as a mean or median value, SOFM-NN for strings have been defined (Kohonen, 1998). These SOFM-NN are organized as a symbol strings array, whereby the relative locations of the strings on the SOFM ought to reflect some distance measure (e.g. LD, FD) between the strings. The idea behind the pure symbolic SOFM-NN is to define similarities or distances between data objects. In our application data objects are represented by symbolic strings. Based on these similarities/distances, finding representative prototypes (for our application, meaningful frescoes) will be the next step.

In training of pure symbolic SOFM-NN, two steps are repeated:

- Find best-matching unit (BMU) for each data item, and add the data item to the list of its best-matching unit; BMU is found using the defined similarity/distance measure,

- Update the models in SOFM nodes: find the median data item belonging to the union of the list (data list union contains all data items in the neighbourhood of the current node being update).

For computing the median data item, assume there are 3 data items (e.g. symbol strings $S_{1}$, $\mathrm{S}_{2}, \mathrm{~S}_{3}$ ) and the following pair wise distances (Table 2):

\begin{tabular}{|c|c|c|c|}
\hline & $\mathrm{S}_{1}$ & $\mathrm{~S}_{2}$ & $\mathrm{~S}_{3}$ \\
\hline $\mathrm{S}_{1}$ & 0 & 4 & 2 \\
\hline $\mathrm{S}_{2}$ & 4 & 0 & 2 \\
\hline $\mathrm{S}_{3}$ & 1 & 2 & 0 \\
\hline
\end{tabular}

Table 2. Pair wise distances.

then compute the sum of the distances from each data item to others:

$$
\begin{aligned}
& S_{1}: 0+4+1=5 \\
& S_{2}: 4+0+2=6 \\
& S_{3}: 1+2+0=3
\end{aligned}
$$

The smallest sum of distances is for data item $\mathrm{S}_{3}$, so that is the median of this data set. In the case of SOFM-NN, the distances could be weighted by the value of neighbourhood function (e.g. a Gaussian-shaped neighbourhood function). 


\section{Experimental results}

\subsection{Application of the resemblance and barycentre criteria in simple environment}

The two criteria apply only on the certain landmarks and have been tested in two types of environments. In a first step, experiments in simple environments led us to point out the thresholds relevant ranges. In a second step, a complex environment has been used to validate these thresholds.

The problem is to find the right threshold for each criterion. A representative panel of situations is first established and systematic tests are made on each situation in which the frescoes are listed for different thresholds of the two criteria. Then a reference threshold for each criterion is fixed taking into account firstly the ratio of kept frescoes and secondly the position of these frescoes with respect to their situation along the robot's route in the considered environment. Finally, thresholds that have been defined are tested in a complex environment.

(a) Choice of different types of environment: indoor environments can be described using a limited number of situations (Al Alan, 1995): openings, walls, angles, room, corridor, deadend and crossings. So far, tested situations are listed in Table 3. Fig. 8 shows the example of the "opening on the left situation". Numbers on the left of the figure show the different positions where frescoes have been constructed. In this example, frescoes are built from position 1 to position 31 (only one of five is drawn to make the figure readable).

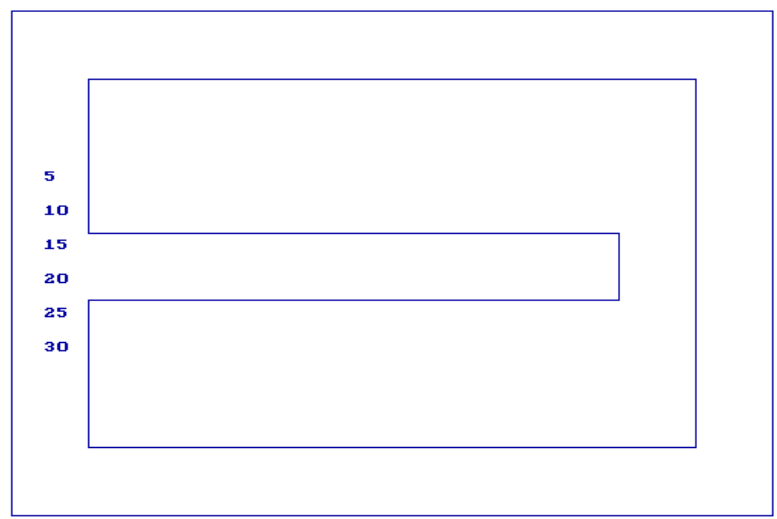

Fig. 8. Example of situation: Opening on the left.

In the different situations, the initial numbers of frescoes are different (Table 3).

\begin{tabular}{|c|c|c|}
\hline & Situation & Number of frescoes \\
\hline Angle to the left & AL & 31 \\
\hline Angle to the right & AR & 31 \\
\hline Opening on the left & OL & 31 \\
\hline Opening on the right & OR & 31 \\
\hline X-crossing & CX & 42 \\
\hline
\end{tabular}

Table 3. Initial number of built frescoes.

(b) Number of pertinent frescoes vs. criterion: it is firstly interesting to observe the number of frescoes kept for different values of thresholds. For barycentre criterion, values between 0 
and 2 with a step of 0.05 are tested. For resemblance criterion, values between 0 and 12 with a step of 0.5 are tested. Beyond these limits, only fresco number one is kept. As the initial number of frescoes is different in all situations, the ratio between the number of frescoes kept and the initial number of frescoes is analysed. Fig. 9 shows the results for resemblance criterion. Fig. 10 shows the results for barycentre criterion. It can be seen that curves in each figure are similar, meaning that criteria have the same response in all the environment situations. It seems then possible to find a common threshold.

\section{Resemblance}

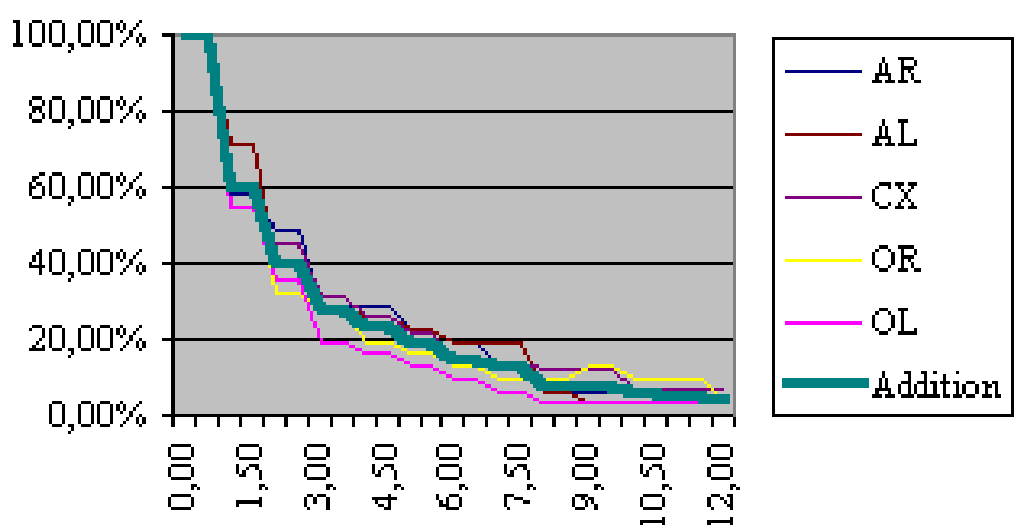

Fig. 9. Percentage of frescoes selected by resemblance criterion vs. threshold value (AR/AL: angle on the right/left, CX: X-crossing, LA: lab, OR/OL opening on the right/left, Sum: add up).

\section{Barycenter}

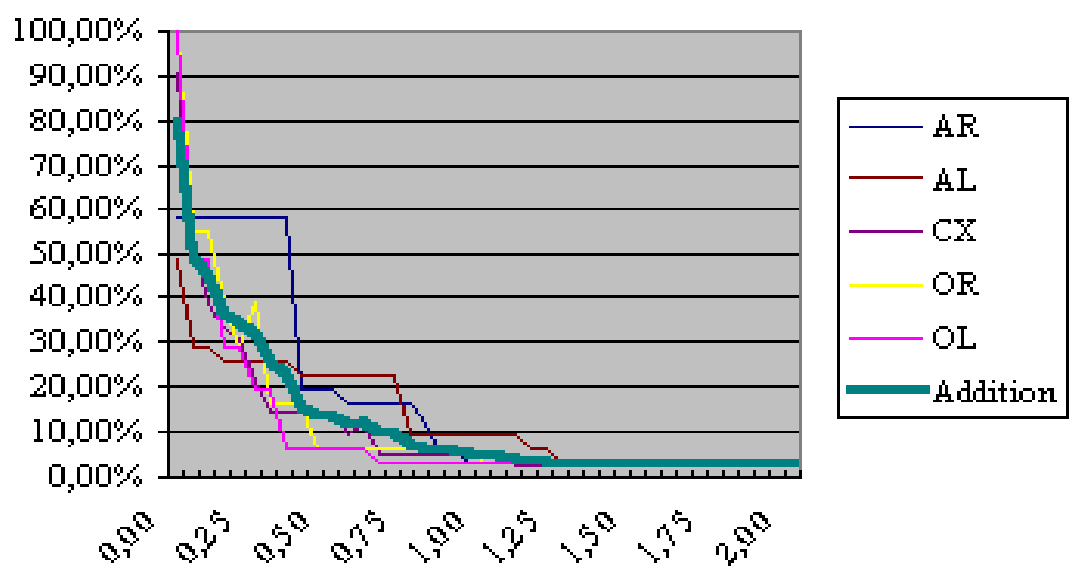

Fig. 10. Percentage of frescoes selected by barycentre criterion vs. threshold value (AR/AL: angle on the right/left,CX: X-crossing, LA: lab, OR/OL opening on the right/left, Sum: add up). 


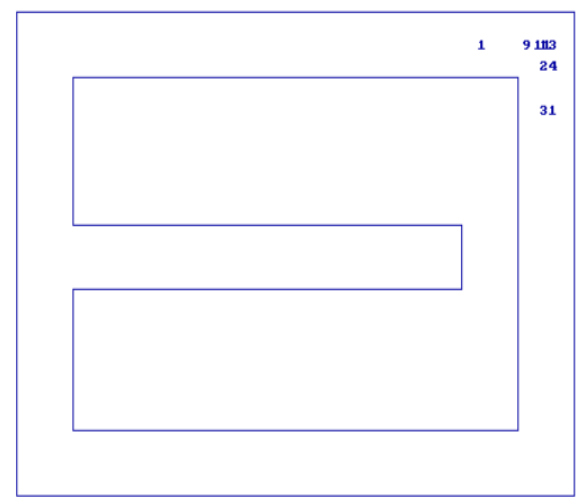

ad - Barycentre 0,40

a)

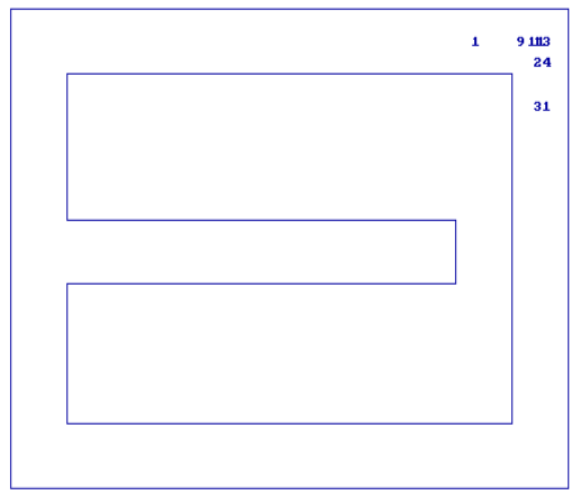

ad - Barycentre 0,50

c)

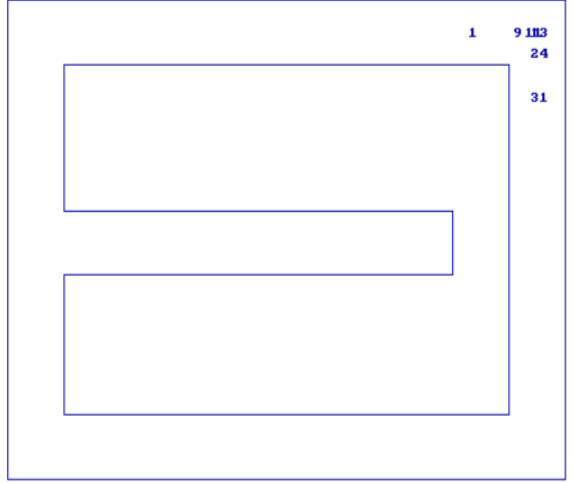

ad - Barycentre 0,45

b)

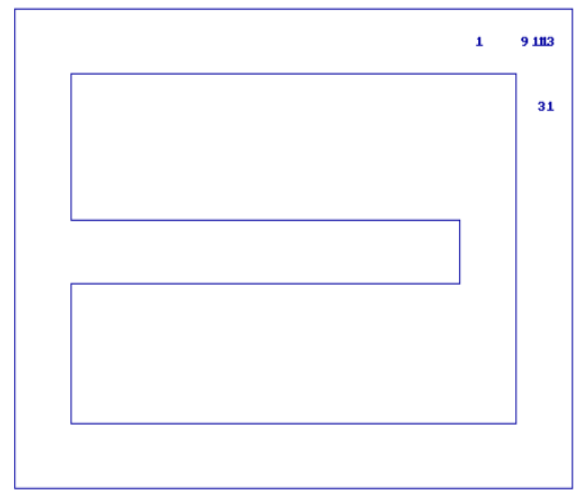

d - Barycentre 0,55

d)

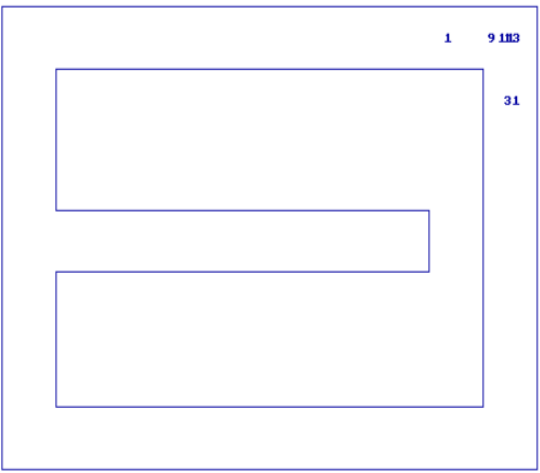

ad - Barycentre 0,60

e)

Fig. 11. Pertinent frescoes vs. barycentre criterion (AL situation); a) threshold $=0.40 ; b$ ) threshold $=0.45 ;$ c) threshold $=0.50 ; \mathrm{d}$ ) threshold $=0.55$; e) threshold $=0.60$. 
It also can be noted that curves decrease quickly for low thresholds values. In fig. 8 , frescoes between 1 and 10 represent the same part of the environment with very slight differences. The objective is to keep a reasonable part of frescoes between $10 \%$ and $20 \%$ in the first approximation. That means thresholds values comprise between 5 and 7 for resemblance criterion and between 0.4 and 0.6 for barycentre criterion.

(c) Positions of pertinent frescoes: for both criteria, it is interesting to visualise which frescoes are considered as pertinent (fig. 11). Frescos number 1 and 31 represent the beginning and the end of the trajectory: they appear for all thresholds. Frescoes 9, 11, 13 and 24 represent the heart of the turning. They are very close considering Euclidean distance but they differ in term of orientation. Fresco number 24 disappears for thresholds equal to 0.55 or 0.60 . The value 0.50 is the central threshold value for barycentre criterion. A similar analysis has been conducted for all other situations. In the same way, the resemblance criterion leads to the same conclusion with 6.0 as central threshold.

\subsection{Application of the resemblance and barycentre criteria in complex environments}

A complete trajectory has been studied in a complex environment (fig. 12 a)). The two criteria have been applied. The variations of the thresholds have been limited to the range determined by the tests in simple environments: 5 to 7 for resemblance and 0.4 to 0.6 for barycentre. Fig. 13 shows the percentage of selected frescoes for both criteria. For barycentre criterion, there is no significant difference between the complex and the simple environments. For resemblance criterion, the ratio is greater in the complex environment than in the simple ones. Nevertheless, for a threshold equal to 7.0, the ratio becomes close to the ratio obtained in simple environments.

\subsection{Application of the other criteria}

Against the frescoes acquired (fig. 12 b)) from the lab environment (Hoppenot, 2003), the above mentioned possibilities of salient frescoes selection were implemented and compared.

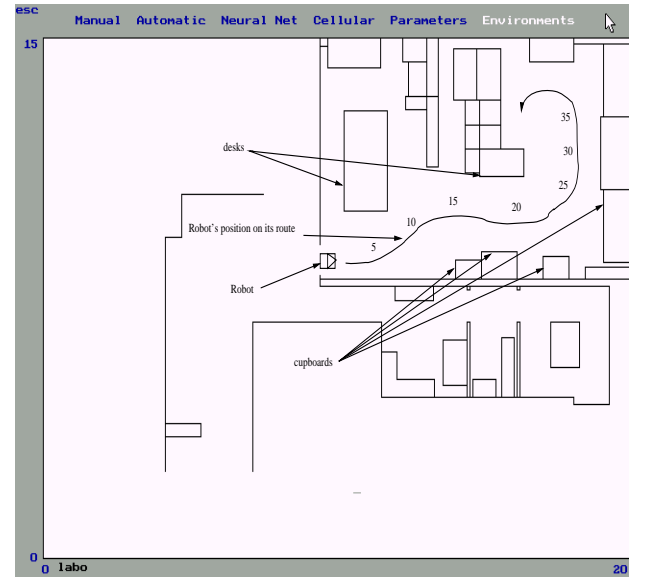

a)

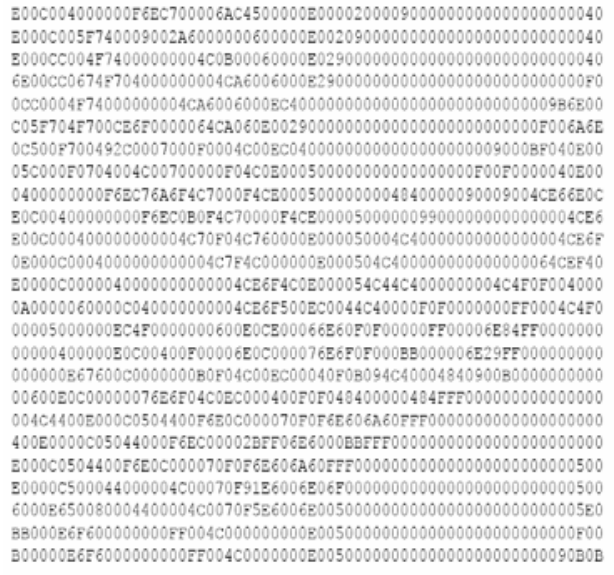

b)

Fig. 12. a) Test environment: the lab; b) Frescoes acquired by the robot from the environment shown in the left. 
Resemblance criterion

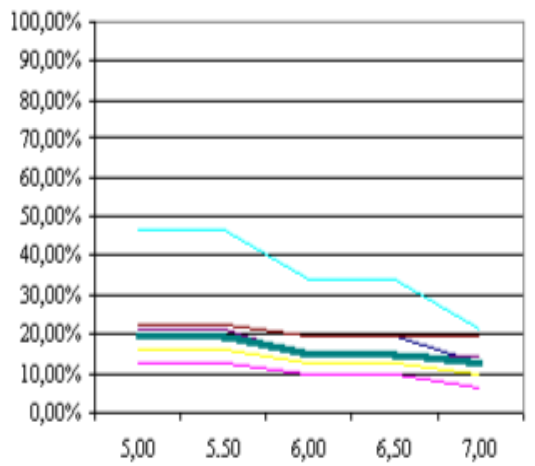

Barycenter criterion

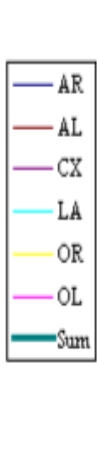

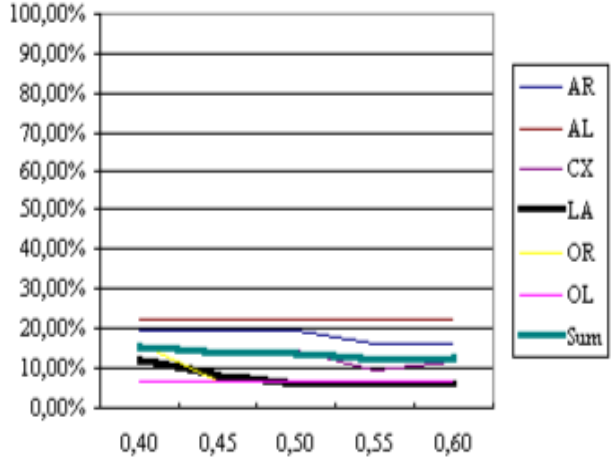

Fig. 13. Comparison of percentage of frescoes selected by resemblance/barycentre criterion in complex (LA) and simple environments vs. threshold.

The current numbers of the selected frescoes are synthetically presented in Table 4 .

\begin{tabular}{|c|l|}
\hline Method & \multicolumn{1}{|c|}{ Index } \\
\hline R & 23822232425 \\
\hline B & 4911132123 \\
\hline H & 9101315171820 \\
\hline C & 91115171922 \\
\hline N & 9111517181925 \\
\hline
\end{tabular}

Table 4. Indexes of selected frescoes with R-Resemblance, B-Barycentre, H-Hamming, LLevenshtein, C-Cross-correlation, N-Neural Network.

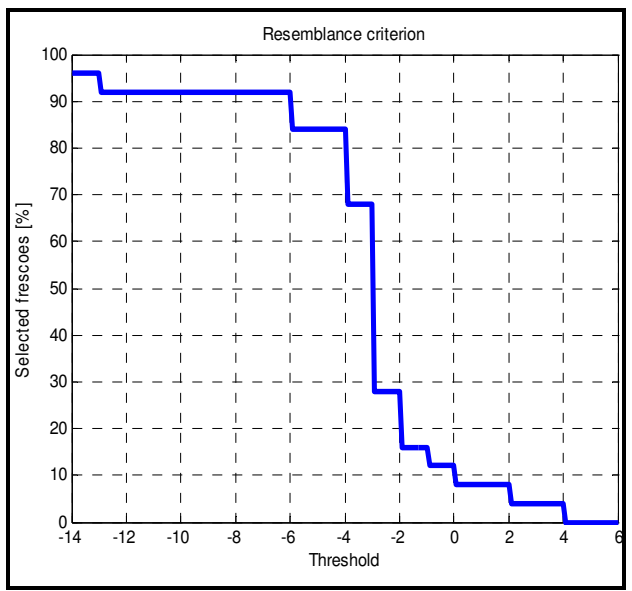

(a)

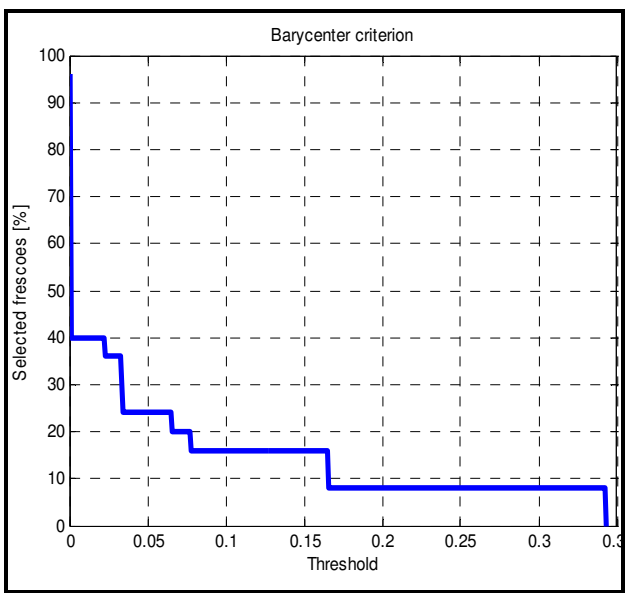

(b) 


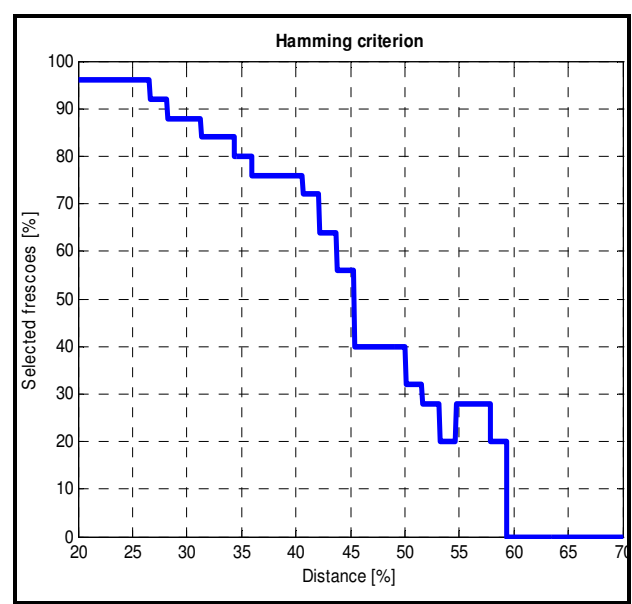

(c)

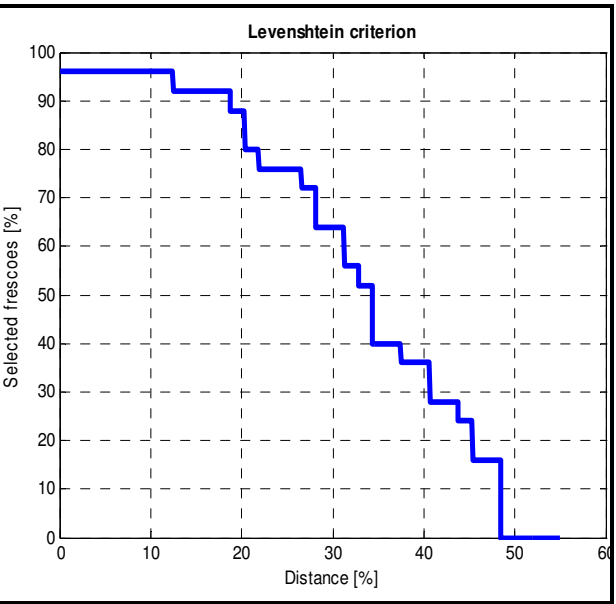

(d)

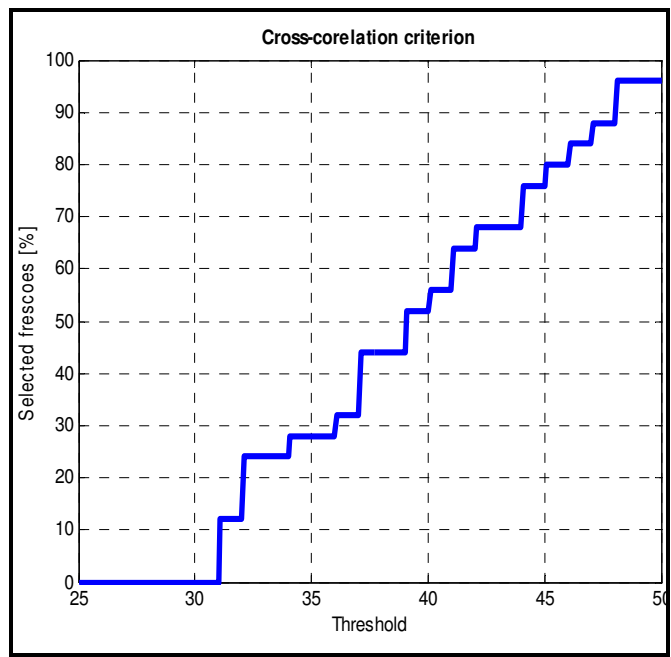

(e)

Fig. 14. The dependence percent of selected frescoes - threshold.
(a) Resemblance criterion;
(b) Barycentre criterion;
(c) Hamming criterion;
(d) Levenshtein criterion
(e) Cross-correlation criterion;

In fig. 14 the dependence percentage of selected frescoes vs. threshold is depicted. Fig. 15 show the salient frescoes selected by each method. An acceptable percent of the selected meaningful frescoes should be around $30 \%$ or less from the total amount of frescoes. In absolute values this mean around 7 frescoes selected. 


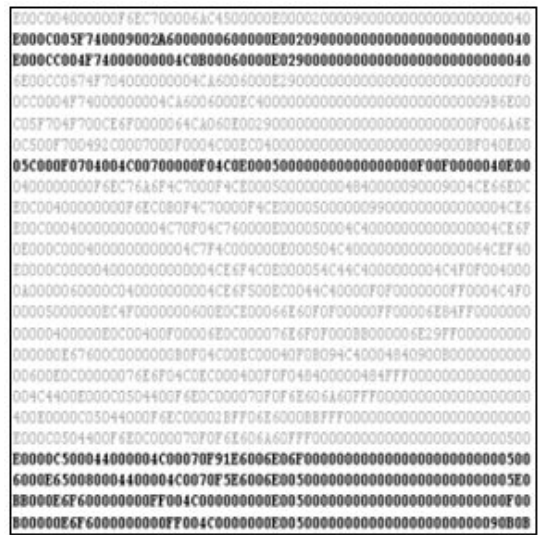

a)

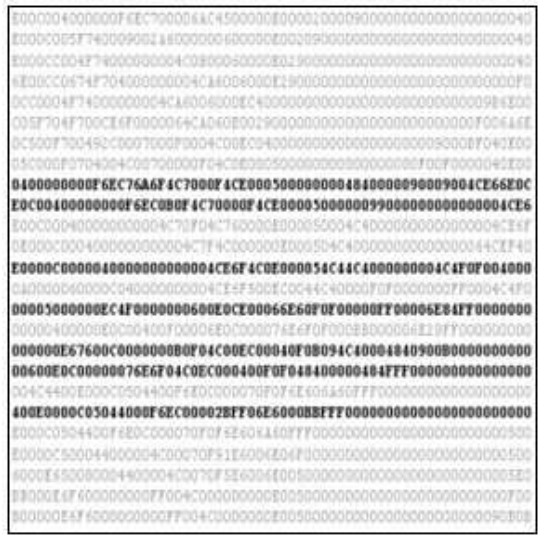

d)

\begin{tabular}{|c|}
\hline 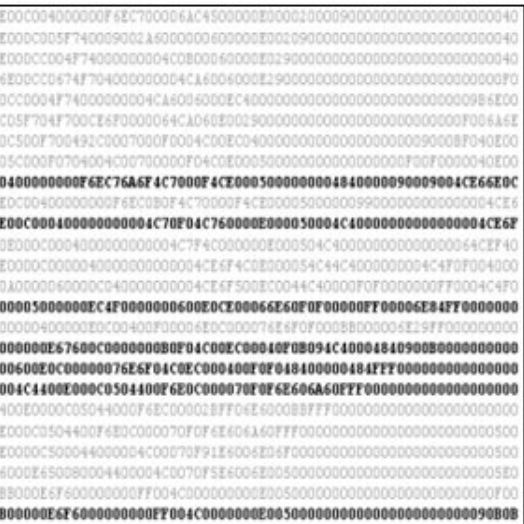 \\
\hline
\end{tabular}

e)

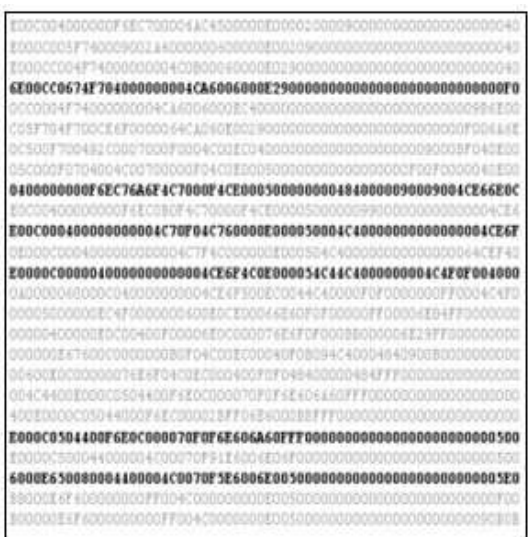

b)

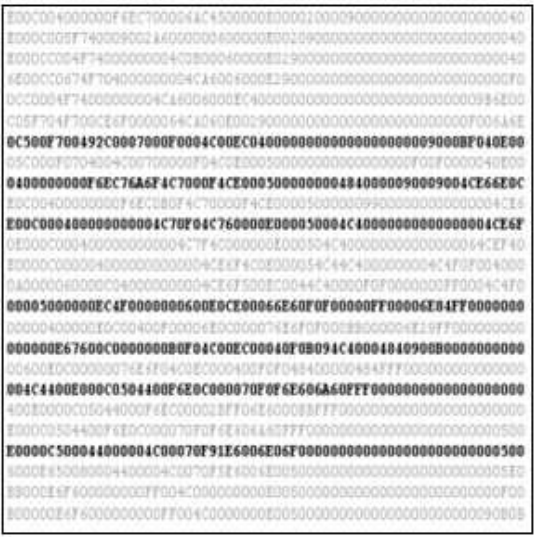

e)

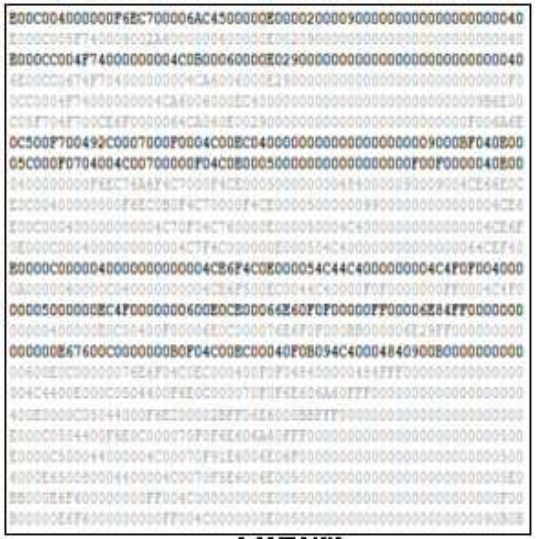

f)

Fig. 15. Selected salient frescoes using the criteria of: a) resemblance; b) barycentre; c) Hamming; d) Levenshtein; e) cross-correlation; f) SOFM-NN. 


\section{Future works and conclusion}

Human beings, as well as insects (Collet \& al., 1992), use resemblance (or dissimilarity) to compare views of the environment rejecting those that do not bring up new elements without using metrics, only using the occurence of landmarks. In this work, we propose a qualitative method inspired of homing methods (Weber, 1999) to construct the environment surrounding an indoor mobile robot equipped with a $2 \mathrm{D}$ telemetry sensor. Every times distance measurements are made, landmarks are extracted and organised into series called frescoes. From this point, distance information are no longer used. In order to derive the pertinent frescoes that can describe the trajectory of the robot, we plan to use a pairing-like method. The first criterion that is primarily being investigated uses a resemblance between two frescoes. The landmarks are bounded and a correlation function measures the difference between consecutives frescoes. The second criterion is based on the difference between the barycentre positions of consecutive frescoes (Huttenlocher, 1993). Those frescoes separated by a difference higher than a threshold are considered as pertinent to describe the robot's route. In both cases the differences are compared with thresholds that are experimentally set up. Despite the criteria simplicity, the results in the very changing test environment (Fig. 12a) show that the thresholds experimentally trimmed in simple environments are well fitted to a complex environment. But the resemblance and barycentre methods have the disadvantage of not taking into account the qualitative aspect of landmarks but only the quantitative one. Lets consider an hypothetically example, in which two consecutive frescoes are completely different but has the same number of landmarks/quadrant. Both methods will give an inappropriate answer, resulting in meaningful frescoes losses, because both operate with number, not type, of landmarks. The value of the selection threshold for the resemblance method is also difficult to be anticipated because of rapid variation of the number of selected frescoes in the region of the optimal threshold. It could be easily observed the poor performance of this criterion: the marked frescoes are somehow similar and not representative. The barycentre method is similar with the previous one: in selecting the salient frescoes only number of landmarks from quadrants are counted. It differs in respect of computing the difference between strings and it seems to give slightly better results.

The Hamming distance compares two strings/frescoes character by character, the distance representing the number of different characters found. Here the selection threshold has been expressed in percentage form. The principle underlying Hamming distance is totally different from the previous two methods: it takes into account the qualitative aspect of strings and, as a consequence, is a better solution. In spite of this fact one might consider it giving unsatisfactory results. Let's take a fragment from two successive frescoes, for example: ... $0004 \mathrm{~F} 74000 \ldots$ and ... $004 \mathrm{~F} 740000 \ldots$ It is clear that these two consecutive frescoes contain basically the same landmark. The 1-character left shift is an environment perspective changing due robot movement along the trajectory. Although, HD score is very high, as the two consecutive frescoes were completely different, resulting in a possible selection of both strings.

This kind of problem is not present in the case of Levenshtein distance. LD computes the distance, in this case, as a simple insertion operation, the distance being kept at minimum. It appears that this method is the best solution for the problem of salient 
frescoes selection. The computationally cost represents the main disadvantage of LD. One might observe that frescoes are padded with lots of zeros representing empty cells. In order to reduce the computation time, these empty spaces might be ignored. We called the result fast Levenshtein distance, fLD, which produce, in terms of selected frescoes, the same results as LD, but in a significantly shorter time. Almost the same results as LD are given by the cross-correlation principle. Due to alignment underling principle of these methods, the perspective modification of landmarks in a fresco is not seen as a fundamental change.

The SOFM-NN implemented has an input layer of 256 (64 symbols/fresco $\times 4$ bits) neurons and 7 output neurons. Thus, the training is constituted of 25 binary vector having 256 elements. The network has been trained for 5000 epochs. After the learning phase, the seven weight vector corresponding to the output neurons should represent the essential frescoes selected from the input set.

Using a SOFM neural network for salient frescoes selection turns out to be improper. Among possible explanations are:

- The reduced size of training elements; The 25 considered set of frescoes are not enough to form appropriate prototype vectors. Thus, prototype vectors are not entire identically with some of the 25 training frescoes.

- There is no sufficiently redundancy in the 25 frescoes selected.

- The conversion process frescoes -> binary vectors -> real numbers and vice-versa generates errors.

Within the framework of mobile robots navigation, six methods for salient frescoes selection were described and tested. Of the six, the Levenshtein distance and cross-correlation defined for strings approaches produced the most accurate results and had some benefits in interpreting the score in meaningful ways (see Table 5). The good results given by these approaches could be explained based on theirs ability in dealing with frescoes perspective modification.

\begin{tabular}{|l|l|l|l|l|l|l|l|}
\hline & $\mathbf{R}$ & $\mathbf{B}$ & $\mathbf{H}$ & $\mathbf{L}$ & $\mathbf{C}$ & $\mathbf{N}$ & Score \\
\hline $\mathbf{R}$ & - & $1(23)$ & 0 & $1(22)$ & $1(25)$ & $2(3,8)$ & 5 \\
\hline $\mathbf{B}$ & $1(23)$ & - & $2(9,13)$ & $2(9,11)$ & $2(9,11)$ & $1(13)$ & 8 \\
\hline H & 0 & $2(9,13)$ & - & $3(9,15,17)$ & $4(9,15,17,18)$ & $3(13,15,17)$ & 12 \\
\hline $\mathbf{L}$ & $1(22)$ & $2(9,11)$ & $3(9,15,17)$ & - & $5(9,11,15,17,19)$ & $3(7,15,17)$ & 14 \\
\hline C & $1(25)$ & $2(9,11)$ & $4(9,15,17,18)$ & $5(9,11,15,17,19)$ & - & $2(15,17)$ & 14 \\
\hline $\mathbf{N}$ & $2(3,8)$ & $1(13)$ & $3(13,15,17)$ & $3(7,15,17)$ & $2(15,17)$ & - & 11 \\
\hline
\end{tabular}

Table 5. Common selected frescoes. Based on these common frescoes a score for each method is computed.

Legend: R-Resemblance, B-Barycentre, H-Hamming, L-Levenshtein, C-Cross-correlation, NNeural Network.

One application field is service robotics (e.g., supplying help to old or handicapped persons.). It can be easily foreseen that the robot will have to make return journeys in the user's flat. The problem is then fourfold: i) the journey must be described using a humanlike language, ii) series of frescoes are inferred from the route description, iii) navigation uses these series to lead the robot to the target point, iv) the robot has to return to its starting point and must retrieve its route using only the pertinent frescoes recorded when on the way on? 
Point 1 was studied in (Saïdi, 2006). From a high-level route description, the robot's journey is built. The problem was extended to a group of robots. To solve point 4 , selected frescoes describing the way on are stored in robot's memory (LIFO). After having been processed its task, the robot has to return on a route that is not exactly the same than the way on. Therefore, the current fresco does not correspond exactly to the stored frescoes (the $180^{\circ}$ rotation is, obviously, taken into account): the fresco and one situated on the top of the LIFO do not correspond. A first method consists in shifting left or right the current fresco to better fit to one of the stored frescoes (Pradel, 2000). Another method consisting in gathering landmarks into representative sets (alcove, cupboard ...) and using all possible transformations of the current fresco is too time consuming. On the contrary, a method grounded on the study of the evolution of very small groups of landmarks is more promising, simple and low resource consuming. On the other hand, with this method, the robot must anticipate the future environments. This anticipation, even if it needs a complete description of all transforms of a fresco, is simpler when the fresco is split into small groups of landmarks. Anticipating frescoes from the current one and comparing them with the stored frescoes seems to be a promising method that will allow the robot to choose the right return way. First results show that the robot is able to return to its starting point in various environments. Nevertheless, the method must be validated in complex and changing environments.

Present and future works focus on points 2, 3. Another perspective is to use a single vision sensor (CCD camera) instead of the laser range finder, extracting distances from images to build a structure similar to frescoes.

\section{References}

Aha, D.W.; Kibler, D. \& Albert, M.K. (1991). Instance-based Learning Algorithms, Machine Learning, vol. 6(1), pp. 37-66, Springer Netherlands, ISSN: 0885-6125, 1991

Ahuactzin, J; E. Mazer, E. \& Bessière, P, (1995). L'algorithme fil d'Ariane, Revue d'intelligence artificielle, vol. 9(1), pp. 7-34, 1995

Al Allan, S.; Pradel, G.; Barret, C. \& Abou Kandil, H. (1995). Neural Architectures for Mobile Robot Navigation, International Conference on Advanced Robotics and Intelligent Automation, pp. 440-445, Athens. Greece, 1995

Altschul, S., (1991). Amino acid substitution matricesfrom an information theoretic perspective, Journal of Molecular Biology, vol. 219, pp. 555-565, 1991

Arkin, G., (1998). Nomad 200 - simulator MissionLab, Behaviour-Based Robotics, MIT Press, 1998

Bras, F.; Pradel, G. \& Jin, Z., (1995). Cellular Automata Applied to the Path Generation and Environment Representation for a Mobile Robot, IFAC Motion Control Conference, pp. 395-402, Munich, Germany, 1995

Blanzieri, E. \& Rizzi,F. (1999). Advanced metrics for class driven similarity search, Proceedings of $10^{\text {th }}$ International Workshop on Database and System Application, pp. 223227, Firenze, Italy, 1999

Choset, H. \& Nagatani, K. (2001). Topological Simultaneous localization and Mapping (SLAM): Toward Exact Localization without Explicit Localization, IEEE Transaction on Robotics and Automation, vol. 2(17), pp. 125-137, 2001

Collet, T.; Dillmann, E.; Giger, A.; Wehner, R. (1992). Visual landmarks and route following in desert ants, Journal of Comparative Physiology, Springler Verlag, vol. 170, pp. 435442,1992 
Crosnier, A. (1999). Modélisation géométrique des environnements en robotique mobile, French Workshop on Robotic Resarch (Journées Nationales de la Recherche en Robotique), Montpellier, France, pp. 83-91, 1999

DeSouza, G.N. \& Kak, A.C. (2002). Vision for mobile robot navigation: a survey, IEEE Transaction on pattern analysis and machine intelligence, Vol. 24(2), pp. 237-267, 2002

Filliat, D. and Meyer, J.A. (2002a). Map-Based Navigation in Mobile Robots. I. A review of Localization strategies, http://citeseer.ist.psu.edu/filliat03mapbased.html

Filliat, D. \& Meyer, J.A. (2002b). Global localization and topological map-learning for robot navigation, $h$ ttp://citeseer.ist.psu.edu/filliat02global.html

Fleisher, J.; Marshland S. \& Shapiro, J. (2003). Sensory anticipation for autonomous selection of robot landmarks, http://www.cs.man.ac.uk $\sim \sim$ fleischj/research.html

Franz, M.; Schölkopf, B. \& Bülthoff, H. (1997). Image-based Homing, Proceedings of the European Conference on Artificial Life, pp. 236-245

Gaussier, P. ; Joulain, C.; S. Zrehen, S. \& Revel, A. (1997). Image-based Homing, Proceedings of the IEEE International Conference on Intelligent Robots and Systems, pp. 545-550, 1997

Gusfield, D. (1997). Algorithms on strings, Trees and Sequences, Computer Science and computational Biology, Cambridge University Press, NY, USA, 1997

Haykin, S. (1999). Neural neworks, A Comprehensive Foundation, second Edition, Prentice Hall, ISBN: 013273350 1, 1999

Hong, I. (1991). Image-based Homing, Proceedings of the IEEE International Conference on Robotics and Automation, Sacramento, USA, pp. 620-625, 1991

Hoppenot, P.; Pradel, G.; Căleanu, C.D.; Perrin, N. \& Sommeilly, V. (2003). Towards a symbolic representation of an indoor environment, Proc. IEEE-SEE-CESA2003 Computing Engineering in Systems Applications, CD ROM paper no. S1-R-00-0048, Lille, France, 2003

Huttenlocher, D.; Klanderman, G. \& Rucklidge, W. (1993). Comparing images using Hausdorff distance, IEEE Transactions on pattern analysis and machine intelligence, vol. 15(9), pp. 850-863, 1993

Judd, S. \& Collett, T. (1998). A Mobile Robot That Learns Its Place, Nature, vol. 392, pp. 710 $714,1998$.

Karlin, S. \& Altschul, S. (1990). Methods for assessing the statistical significance of molecular sequence features by using general scoring schemes, proceedings of the national Academy of Science, vol. 87, pp. 2264-2268, 1990

Kim, D. \& Neviata, R. (1994). A method for recognition and localization of generic objects for indoor navigation, Proceedings of ARPA Image Understanding Workshop, Monterey, USA, 1994

Kohonen, T. (1987). Content-Addressable Memories, Springer Series in Information Sciences, vol. 1, Springer Berlin Heidelberg, 1987

Kohonen, T. (1988). Self-Organization and Associative Memory, Springer Series in Information Sciences, vol. 8, Springer Berlin Heidelberg, 1988

Kohonen, T. \& Somuervo, P. (1998). Self-organizing maps of symbols strings, Neurocomputing, vol. 21, pp. 19-30, Elsevier, ISSN: 0925-2312, 1998

Kuipers, B. \& Byan, Y.T. (1991). A robot exploration and mapping strategy based on a semantic hierarchy of spatial representation, International Journal of Autonomous Systems, vol. 8, pp. 47-63. 
Kulic, R. and Vukic, Z. (2003). Methodology of concept control synthesis to avoid. unmoving and moving obstacles, The Knowledge Engineering Review, Vol. 18, issue 3, pp. 281-291, Cambridge University Press

Lamon, P.; Nourbakhsh, I.; Jensen, B. \& Siegwart, R. (2001). Deriving and Matching Image Fingerprint Sequences for Mobile Robot Localization, http://citeseer.nj.nec.com/445679.html, Proceedings of the IEEE International Conference on Robotics and Automation, Seoul, Korea, 2001

Leonard, J. J. \& Durrant-Whyte, H.F. (1991). Simultaneous map building and localization for an autonomous mobile robot, Proceedings of the IEEE/RSJ Int. Workshop on Intelligent Robots and Systems IROS'91, pp. 1442-1447, New York, NY, USA, 1991

Levenshtein, L.I. (1966). Binary codes capable of correcting deletions, insertions, and reversals, Soviet Physics-Doklady, vol. 10, no.7, pp. 707-710, 1966.

MacKenzie, P. \& Dudek, G. (1994). Precise Positioning using Model-Based Maps, Proceedings of IEEE International Conference on Robotics and Automation, pp. 1615-1621, San Diego, USA, 1994

Meyer, J.A. \& Filliat, D. (2002). Map-based navigation in mobile robots - II. A review of map-learning and path-planning strategies, http://citeseer.ist.psu.edu/meyer03mapbased.html

Oore, S.; Hinton, G. \& Dudek, G. (1997). A Mobile Robot That Learns Its Place, Neural Computation, pp. 683-699, MIT Press, vol. 9(3)

Pomerleau, D.A. (1993). Neural Network Perception for Mobile Robot Guidance\}, Kluwer Academic Publishers.

Pradel, G.; Bras, F. \& Jin, Z. (1994). 2D laser telemetry-based path trend generation and real time environment symbolic representation for an autonomous mobile robot,

Proceedings of the IFAC-IEEE International Conference on Machine Automation, pp. 122-134, Tampere, Finland, 1994

Pradel, G.; Avrillon, S. \& Garbuio, L. (2000). Landmark interpretation by means of frescoes in mobile robotics, Proceedings of the 6th Int. Conf. On Methods and Models in Automation and Robotics\}, pp. 585-592, Miedzyzdroye, Poland, 2000.

Pradel, G. \& Bras, F. (2001). Qualitative environment description by means of frescoes in mobile robotics, Journal européen des systèmes automatisés, vol. 9(35), pp. 1105-1128, ISSN 1269-6935, 2001.

Saïdi, F. \& Pradel, G. (2006). A multi-robot path planner for group navigation, Journal of Intelligent and Robotics Systems, Springer-Verlag eds, under press, 2006

Simhon, S. \& Dudek, G. (1998a). A global Topological Map formed by Local Metric Maps,

Proceedings of IEEE/RSJ International Conference on Intelligent Robotic Systems, Victoria, B.C., Canada, pp. 1708--1714, 1998

Smith, C.M. \& Leonard, J.J. (1997). A multiple-hypothesis approach to concurrent mapping and localization for autonomous underwater vehicles, Proceedings of International Conference on Field and Service Robotics, pp.249-256, Canberra, Australia, 1997

Tedder, M. \& Hall, L.E. (2001). Symbolic processing methods for 3D visual processing, http://www.robotics.uc.edu/papers2001/Maurice2001d.pdf

Thrun, S. (2002). Robotic mapping: A survey, Exploring Artificial Intelligence in the New Millenium, Morgan Kaufmann, http://citeseer.ist.psu.edu/thrun02robotic.html

Wagner, R.A. \& M. J. Fischer, M.J. (1974). The string to string correction problem, Journal of the ACM, 21, pp. 168-173, 1974. 
Weber, K.; Venkatesh, S. \& Srinivasan, M.V. (1999). Insect Inspired Robotic Homing, Adaptive Behavior, vol. 1, pp. 65-98

Wichert, G. (1996). Selforganizing Visual Perception for Mobile Robot Navigation, http://www.rt.e-technik.th-darmstadt.de/ georg/pubs/EUROBOTS96/paper.html 


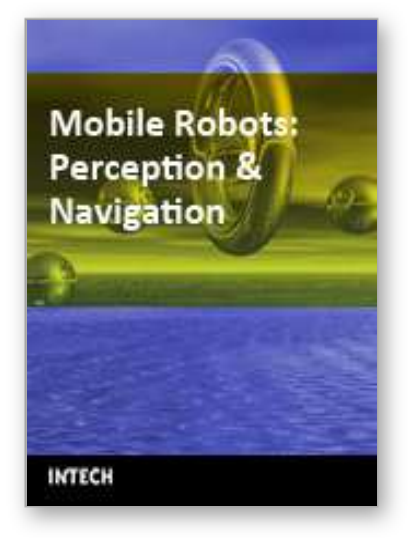

\author{
Mobile Robots: Perception \& Navigation \\ Edited by Sascha Kolski
}

ISBN 3-86611-283-1

Hard cover, 704 pages

Publisher Pro Literatur Verlag, Germany / ARS, Austria

Published online 01, February, 2007

Published in print edition February, 2007

Today robots navigate autonomously in office environments as well as outdoors. They show their ability to beside mechanical and electronic barriers in building mobile platforms, perceiving the environment and deciding on how to act in a given situation are crucial problems. In this book we focused on these two areas of mobile robotics, Perception and Navigation. This book gives a wide overview over different navigation techniques describing both navigation techniques dealing with local and control aspects of navigation as well es those handling global navigation aspects of a single robot and even for a group of robots.

\title{
How to reference
}

In order to correctly reference this scholarly work, feel free to copy and paste the following:

Pradel Gilbert and Caleanu Catalin Daniel (2007). Symbolic Trajectory Description in Mobile Robotics, Mobile Robots: Perception \& Navigation, Sascha Kolski (Ed.), ISBN: 3-86611-283-1, InTech, Available from: http://www.intechopen.com/books/mobile_robots_perception_navigation/symbolic_trajectory_description_in_m obile_robotics

\section{INTECH}

open science | open minds

\section{InTech Europe}

University Campus STeP Ri

Slavka Krautzeka 83/A

51000 Rijeka, Croatia

Phone: +385 (51) 770447

Fax: +385 (51) 686166

www.intechopen.com

\section{InTech China}

Unit 405, Office Block, Hotel Equatorial Shanghai

No.65, Yan An Road (West), Shanghai, 200040, China

中国上海市延安西路65号上海国际贵都大饭店办公楼 405 单元

Phone: +86-21-62489820

Fax: $+86-21-62489821$ 
(C) 2007 The Author(s). Licensee IntechOpen. This chapter is distributed under the terms of the Creative Commons Attribution-NonCommercialShareAlike-3.0 License, which permits use, distribution and reproduction for non-commercial purposes, provided the original is properly cited and derivative works building on this content are distributed under the same license. 